\title{
Self-navigation put to asteroid fly-by test
}

[WASHINGTON] A five-minute flight this week by a spacecraft past a tiny, undistinguished asteroid, while not necessarily a great leap for science, could be important for the future of plans by the US space agency NASA to explore the Solar System.

If all goes well, the spacecraft Deep Space 1 (DS1) will today (29 July) pass within 15 kilometres of the centre of the asteroid Braille, far closer than any spacecraft has ever come to a body in the Solar System without landing on it. On-board autonomous navigation, or 'auto-nav', software will direct the 56,000-km-per-hour fly-by with no help from ground controllers, updating the spacecraft's position based on rapidly changing pictures of the target, commanding manoeuvres and deciding when to take photographs.

Since its launch last October, DS1 has shown that it can steer itself during the long cruise phase of a mission using parallax measurements of known asteroids against the star background. The benefit to NASA of this kind of 'hands-off' operation is that the expensive and overbooked Deep Space Network of large radio antennas can be less involved in the routine tracking of spacecraft, and control teams on the ground can be smaller.

The pay-off for science from this week's fly-by will be relatively meagre: around seven black-and-white images with 10metre resolution, and perhaps 30 more with lower resolution, along with limited infrared imagery and data on the plasma environment surrounding Braille.

\section{Scaling the degree of danger from an asteroid}

How worried should we be if astronomers were to report finding an asteroid that could collide with Earth 50 years from now? Planetary scientist Richard Binzel of the Massachusetts Institute of Technology has devised the Torino Impact Hazard Scale as a handy tool for scientists, the media and a public confused by a number of recent false alarms (see Nature 392, 215; 1998)

Similar to the Richter scale used to rank earthquakes, Binzel's scale plots the energetic 'wallop' packed by an incoming asteroid against the likelihood that it will hit Earth, and assigns numerical values ranging from zero (virtually no chance of damage) to ten
But the idea behind the DS1 mission the first in NASA's New Millennium line of spacecraft - is to demonstrate key technologies such as auto-nav so that more scienceorientated missions can incorporate them without being seen as too risky. "We can use it without getting 'dinged' on proposals," says veteran planetary scientist Michael Belton of the National Optical Astronomy Observatories in Tucson, Arizona.

Not everyone in the space science community is convinced of the value of the New Millennium project. It would be better to introduce technological innovation on real, operational science missions, says Stamatios Krimigis, who heads the space science department at Johns Hopkins University's Applied Physics Laboratory (APL), the only institution outside NASA with a major role in managing planetary missions. "Personally, I was never really impressed with demonstrating technology for technology's sake," he says.

With a price tag of $\$ 152$ million, DS1 is not much cheaper than APL's \$211-million Near Earth Asteroid Rendezvous (NEAR) science mission, which will orbit the asteroid Eros for months, mapping its surface at 3metre resolution and returning data on its bulk properties, surface composition and mineralogy across a wide spectral range.

Another Discovery-class mission, the Comet Nucleus Tour (CONTOUR), will fly past three comet nuclei, mapping their surfaces with resolutions down to 4 metres and gathering detailed data on the surrounding

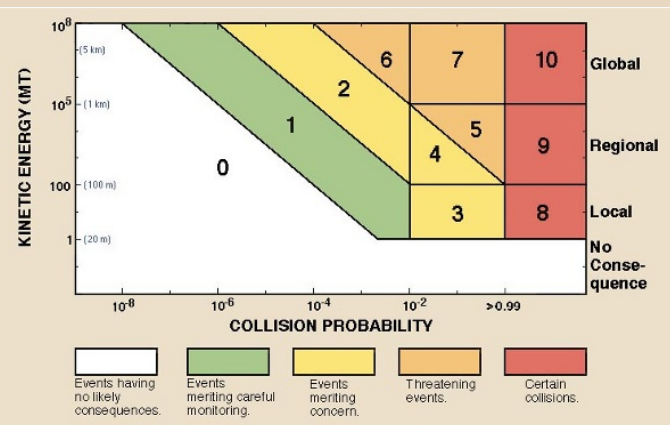

Catastrophe or no? Binzel's Impact Hazard Scale will tell us. (certain catastrophe) to each event.

Adopted last month at a workshop of the International Astronomical Union in Torino, Italy, the idea was endorsed last week by the full union.

The reviews so far have been good. Carl Pilcher, head ofNASA's planetary science programme, called it "a major advance in our ability to explain the hazard posed by a particular object". Binzel hopes his scale will bring discipline to the reporting of impact hazards. "Scientists haven't done a very good job of communicating to the public," he says.

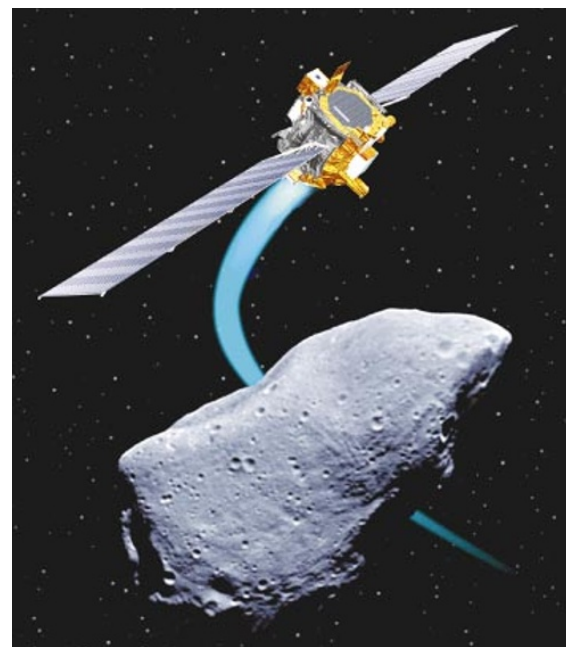

Close call: artist's impression of Deep Space 1's planned encounter with the asteroid Braille.

gas and dust, at a cost of $\$ 154$ million. CONTOUR will also reduce its reliance on the Deep Space Network by going into a spinstabilized 'hibernation' mode during its interplanetary cruise phase.

But even sceptics agree that DS1's successful in-space validation of an ion-drive propulsion engine is an important contribution, as it will make possible certain kinds of spacecraft mission, such as orbiting Jupiter's moon Europa or landing on comet nuclei, that would be impossible or prohibitively expensive with a conventional rocket.

NASA would have been unlikely to approve a scientific mission to an asteroid wouldn't choose a target this small and this poorly known, or go in this close," says Marc Rayman, deputy manager for the mission at the Jet Propulsion Laboratory in Pasadena, California.

Rayman says this week's manoeuvre is "very, very challenging, and it may not work". The approach distance is similar to that of an aircraft flying over the Earth's surface. The spacecraft will not be able to see the mile-wide asteroid until a day before the encounter, and its image will be smaller than one pixel in the camera's view until about an hour before the approach.

By comparison, when the Galileo Jupiter spacecraft flew past two larger asteroids in the early 1990s, scientists on the ground had time to study the approach pictures, plan the photography in advance and radio all the necessary commands to the spacecraft.

If the autonomous fly-by is successful, DS1 managers have an extended mission up their sleeves, should NASA choose to fund it, which would include encounters with two comets: Wilson-Harrington in January 2001, and Borrelly the following September. with DS1's degree of difficulty. "They

Tony Reichhardt 